\title{
ORIGINAL
}

\section{EXPECTATIVAS Y NECESIDADES DETECTADAS EN PROFESIONALES DE ORGANIZACIONES PROVINCIALES QUE TRABAJAN EN ACCIÓN INTERSECTORIAL EN SALUD}

\author{
Mónica Y Martín Agudo (1), José M Sierra Quesada (2), Juan M Jiménez Martín (3) y Cecilia \\ Escudero Espinosa (3)
}

(1) Delegación Provincial de la Consejería de Salud en Granada.

(2) Hospital Universitario "San Cecilio" de Granada.

(3) Escuela Andaluza de Salud Pública.

\section{RESUMEN}

Fundamentos: La Acción Intersectorial en Salud (AIS) ha sido la metodología propuesta para la reducción de la desigualdad en salud. Los objetivos del presente estudio son conocer las experiencias, expectativas y necesidades percibidas de los profesionales que trabajan en organizaciones provinciales, para proponer un marco de análisis y desarrollo de trabajo intersectorial

Métodos: Con un diseño cualitativo se realizaron 21 entrevistas individuales semiestructuradas durante el mes de julio de 2005 a profesionales de organizaciones provinciales de justicia, educación, salud, igualdad y bienestar social y de fuerzas y cuerpos de seguridad en Granada y Sevilla. Se utilizó un muestreo intencional «en bola de nieve» en el que a través de dos informantes clave se logró completar el cuadro de perfiles.

Resultados: Los profesionales identifican necesidades que habrían de ser cubiertas con el trabajo intersectorial así como los fundamentos del mismo. Destacan una serie de características personales y algunas relacionadas con la organización e identifican factores que han sido divididos en políticos, sociales y operativos.

Conclusiones: Hay un consenso en señalar la acción intersectorial como garante para reducir la desigualdad social, ya que evita la duplicidad de recursos y unifica la respuesta que se ofrece. Resulta básico abordar cambios profundos previos en la cultura de las organizaciones provinciales para que el trabajo intersectorial forme parte de las mismas. Tales cambios han de ir acompañados de directrices políticas claras en los niveles superiores y de movimientos sociales que visibilicen la necesidad de una determinada acción intersectorial.

Palabras clave: Acción intersectorial. atención integral de salud, organización y administración, organizaciones y profesionales.

Correspondencia:

Mónica Y Martín Agudo

Carretera Granada, 12.

Pinos Puente. 18240 Granada

Correo electrónico: monica.martin@juntadeandalucia.es
ABSTRACT

\section{Expectations and Needs Detected among Professionals at Provincial Organizations Working in Intersectorial Action in Health}

Background: Intersectorial Action in Health has been the methodology proposed for reducing inequality in health. The objectives of this study are to ascertain what experiences, expectations and needs are perceived to exist among the professionals working at provincial organizations in order to propose a framework for analyzing and carrying out intersectorial work.

Methods: With a qualitative design, 21 semi-structured individual surveys were conducted in July 2005 of professionals from provincial justice, education, health, equality and welfare organization and from law enforcement officials in Granada and Seville. An intentional «snowball» sampling was used, in which it was possible to complete the profile table by way of two individuals answering the survey.

Results: The professionals identified needs which it would be necessary to cover with the intersectorial work as well as the grounds for the same. They highlighted a number of personal traits and some organization-related ones and identified factors which have been divided among, political, social and operative factors.

Conclusions: A consensus exists as to pointing out intersectorial action as a way of guaranteeing the reduction of social inequality, given that it prevents doubling resources and unifies the response being provided. It was thought to be fundamental for some profound changes to first be made in the provincial organizations culture in or for the intersectorial work to comprise part thereof. These changes must go along with clear-cut guidelines at the upper levels of social movements which will clearly highlight the need for a certain intersectorial action in particular.

Key words: Comprehensive health care. Organization and administration. Organizations and professionals review. 


\section{INTRODUCCIÓN}

La definición de la OMS de salud en 1948 como «el estado completo de bienestar físico, mental y social, y no solamente la ausencia de afecciones o enfermedades», marcó un hito en su momento pero en la actualidad ha sido abandonada por las posturas maximalistas y utópicas que proponía. Hoy día se ha adoptado el concepto dinámico de la salud en el que destaca la influencia de los factores sociales ${ }^{1}$. A partir de este enfoque Salleras propone definir la salud como «el logro del más alto nivel de bienestar físico, mental y social, y de capacidad de funcionamiento, que permitan los factores sociales en los que viven inmersos el individuo y la colectividad» ${ }^{2}$. La promoción de la modificación de estos factores sociales ha sido siempre y lo sigue siendo en la actualidad uno de los objetivos más importantes de la salud pública.

Los determinantes de la salud más importantes están relacionados con las condiciones de vida, los factores medioambientales, estilos de vida y factores biológicos tales como la edad, el sexo y la herencia. Desde el enfoque de sistemas, si el estado de salud de las poblaciones son las salidas, las entradas al sistema son las interrelaciones de estos determinantes de la salud ${ }^{3}$.

El término sector ha sido utilizado comúnmente para identificar distintas esferas de la sociedad. Frecuentemente se asume la existencia de dos macrosectores: el económico o sector productivo y el social, y se distingue además al sector político-militar y al científico- tecnológico ${ }^{4}$. Asumiendo esa división de la sociedad, la Acción Integrada de Salud (AIS) puede producirse entre sectores pertenecientes a un mismo macrosector o a distintos macrosectores aunque en la realidad las fronteras de competencia y acción de los diferentes sectores no son tan precisas y varían a lo largo del tiempo o según el contexto incluso dentro de un mismo país ${ }^{4}$.
Se concibe la AIS como la intervención coordinada de instituciones representativas de más de un sector en acciones destinadas total o parcialmente a abordar un problema vinculado con la salud ${ }^{4-7}$.

El concepto de AIS fue introducido en la Declaración de Atención Primaria de Salud en Alma Ata, en $1978^{8}$. Un año después en la formulación de estrategias con el fin de alcanzar salud para todos en el año $2000^{9}$, se introducían la tres dimensiones fundamentales de la AIS: voluntad política, intrasectorialidad o dimensión vertical (niveles de decisión dentro de un mismo sector) e intersectorialidad o dimensión horizontal (relaciones entre sectores dentro del mismo nivel $)^{4,5}$.

La acción intersectorial en salud ha sido la metodología propuesta para la reducción de desigualdades en salud, llegando incluso a proponerse la creación de estructuras específicas ${ }^{10}$.

El Programa de Salud $21^{11}$ en la actualidad supone la respuesta de la Región Europea de la OMS a la política global de «Salud para Todos en el siglo XXI», aprobada por la comunidad mundial en mayo de 1.998. Salud 21 se fundamenta en cuatro estrategias principales de acción de las que una se refiere a la AIS: «Estrategias multisectoriales para hacer frente a los determinantes de la salud, teniendo en cuenta las perspectivas culturales, sociales, económicas, físicas y de género, y garantizando la utilización de la evaluación del impacto sanitario»

La operativización de la AIS podría verse afectada en gran medida por el papel clave que juegan las organizaciones y las profesiones. Las profesiones dominan la definición de los problemas y necesidades sociales y las actuaciones para hacerles frente, y las organizaciones son los espacios que articulan dichos procesos de definición y actuación siendo también utilizadas cuan- 
do los grupos de intereses y las clases sociales realizan acciones colectivas ${ }^{12}$. Desde esta perspectiva, entre los requerimientos de AIS en los distintos niveles de decisión y la sociedad, resulta fundamental conocer el rol e intereses que tienen los grupos profesionales que trabajan dentro de las organizaciones.

Los Planes de Salud en Andalucía ${ }^{13}$, como marco de las políticas de salud en el ámbito autonómico, se han orientado en su evolución hacia la descentralización de las actuaciones. Concretamente los planes provinciales de salud, constituyen documentos de finalidad eminentemente práctica, que orientan e impulsan las políticas de salud en cada una de las provincias andaluzas. Sobre las instituciones provinciales recae el doble reto de reorientar los servicios por un lado hacia los problemas prevalentes en su provincia con eficacia y por otro, sumar y organizar las iniciativas de otros sectores, incluidas las asociaciones y empresas con objetivos de Salud Pública con la mayor eficiencia $^{14}$.

Los ejes sobre los que se han fundamentado los planes provinciales son: a) la lucha contra las desigualdades, como fundamento de la política pública manifestada por la Junta de Andalucía ${ }^{13}$ y b) la intersectorialidad, como elemento imprescindible de la metodología de trabajo de las estrategias transversales que implican las de salud $^{13}$.

\section{OBJETIVOS GENERALES:}

- Conocer las experiencias, expectativas y necesidades percibidas de los profesionales que desarrollan o han desarrollado trabajo intersectorial en el nivel provincial.

- Proponer un marco de análisis y desarrollo de trabajo intersectorial en organizaciones provinciales.

\section{Los OBJETIVOS ESPECÍFICOS son:}

- Explorar las expectativas profesionales, personales, sobre las organizaciones en las que actúan y en cuanto a las poblaciones sobre las que actúan.

- Explorar los fundamentos que los profesionales encuentran para su desarrollo.

- Explorar las necesidades de tipo laboral/profesional y de tipo personal requeridas.

- Identificar factores políticos y sociales que condicionan la respuesta intersectorial en el nivel provincial, así como factores de tipo operativo que facilitan la puesta en marcha de acciones intersectoriales.

\section{SUJETOS Y MÉTODOS}

El estudio se realizó con un diseño cualitativo ${ }^{15}$ y la técnica de recogida de datos utilizada fue la de entrevistas individuales semiestructuradas, realizando un total de $21^{16}$.

Los sujetos de estudio eran profesionales de organizaciones provinciales de los sectores de salud, igualdad y bienestar social, justicia, seguridad y educación; que habían participado anteriormente o en la actualidad en acciones intersectoriales en Granada y Sevilla.

Se utilizó un muestreo intencional «en bola de nieve» en el que a través de dos informantes clave se logró contactar con la mayoría de los participantes. Los primeros entrevistados posibilitaron el contacto con otros profesionales de esas organizaciones completando el cuadro de perfiles. La segmentación se realizó de acuerdo al marco teórico, los objetivos del proyecto y los criterios de homogeneidad, heterogeneidad y exclusión que se exponen a continuación: 


\section{Criterios de homogeneidad}

Profesionales incluidos en el estudio que cumplían las condiciones establecidas dentro del perfil. Se segmentó por tipo de profesional y por sectores. En el tipo de profesional se distinguieron dos perfiles diferentes: 1) Directivos o cargos de responsabilidad y 2) Técnicos

Los sectores sobre los que se diseñaron los perfiles eran: 1) Salud: Personal de la Delegación Provincial de Salud o de cualquiera de los niveles asistenciales, primaria y especializada; 2) Social: Personal de la Delegación de Igualdad y Bienestar Social así como de los Centros de Servicios Sociales Comunitarios de los distintos Ayuntamientos y Diputación Provincial; 3) Educación: Profesionales de la Delegación Provincial de Educación así como profesores de primaria, secundaria o educación especial; 4) Seguridad: Cuerpos de seguridad (policía local o guardia civil), técnicos y cargos de este sector; 5) Judicial: Profesionales que desempeñan sus funciones en las Delegaciones Provinciales de Justicia y Administración Pública así como del Instituto de Medicina Legal dependiente de las mismas.

\section{Criterios de heterogeneidad}

Características que definieron las diferencias entre los grupos a investigar y que aportaron diversidad de información. La finalidad de contemplarlas era identificar discursos posibles y complementarios que hicieran llegar a un modelo integrado en el que cada perfil aportase algún elemento a considerar: experiencia laboral en trabajos intersectoriales de más de 10 años o experiencia de menos de 10 años; ser hombre o mujer; Ámbito habitual de trabajo local, provincial o regional; y tipo de trabajo intersectorial, en el que se determinaron tres niveles teóricos de regulación (volunta- rio o surgido a partir de iniciativas profesionales; normalizado o incluido en las rutinas de trabajo; o regulado, para el que existía una norma de cualquier rango que lo regulaba).

\section{Criterios de exclusión}

Profesionales no contemplados para el estudio bien porque se consideró que restaban factibilidad y no por no considerarlos ámbitos importantes, o bien porque se entendió que no habían tenido suficiente tiempo para tener elaborado un discurso acerca de la intersectorialidad.

Partiendo de los criterios de segmentación y teniendo en cuenta los atributos de los participantes se realizaron 21 entrevistas con los perfiles que figuran en la tabla 1.

\section{Análisis de la información}

Las entrevistas fueron grabadas y transcritas literalmente. La información fue categorizada y analizada mediante el soporte informático Nudist Vivo.

A partir de las preguntas y del objetivo del estudio se elaboraron unas categorías preliminares de análisis que sufrieron modificaciones a lo largo del estudio. Tras la definición exacta y definitiva de las categorías de análisis se procedió a realizar la categorización y codificación en cada uno de los textos de entrevista. A continuación las búsquedas de temas fueron realizadas en función del objetivo del estudio para lo que las categorías fueron agrupadas del modo que figura en la tabla 2.

Se realizó un análisis de contenido de nivel semántico ${ }^{17}$ para cada una de las categorías de análisis agrupadas y relacionadas con el objetivo del estudio. 
Tabla 1

Perfiles de participantes y atributos

\begin{tabular}{|c|c|c|c|c|c|c|c|}
\hline Perfil & Sector & Profesional & Titulación & Experiencia & Trabajo & Ámbito & Sexo \\
\hline RE-16 j & Educación & Responsable & Pedagogo & Más 10 años & Voluntario & Provincial & Hombre \\
\hline RE-1 p & Educación & Responsable & Maestro & Más 10 años & Regulado & Provincial & Hombre \\
\hline RJ-08 e & Justicia & Responsable & Médica & Más 10 años & Voluntario & Provincial & Mujer \\
\hline RJ-18 s & Justicia & Responsable & Abogada & Menos 10 años & Normalizado & Provincial & Mujer \\
\hline RSa-12 I & Salud & Responsable & Médico & Más 10 años & Regulado & Regional & Hombre \\
\hline RSa-21 p & Salud & Responsable & Economista & Más 10 años & Regulado & Regional & Hombre \\
\hline RSe-10 ta & Seguridad & Responsable & $\begin{array}{c}\text { Ingeniero } \\
\text { técnico }\end{array}$ & Más 10 años & Regulado & Provincial & Hombre \\
\hline RSe-15 tr & Seguridad & Responsable & $\begin{array}{l}\text { Ciencias } \\
\text { políticas }\end{array}$ & Menos 10 años & Normalizado & Provincial & Hombre \\
\hline RSo-03 e & Social & Responsable & Pedagoga & Más 10 años & Regulado & Provincial & Mujer \\
\hline RSo-04 m & Social & Responsable & $\begin{array}{l}\text { Trabajadora } \\
\text { social }\end{array}$ & Más 10 años & Normalizado & Local & Mujer \\
\hline RSo-20 r & Social & Responsable & $\begin{array}{c}\text { Trabajadora } \\
\text { social }\end{array}$ & Más 10 años & Normalizado & Provincial & Mujer \\
\hline TE-09 m & Educación & Técnico & Logopeda & Más 10 años & Normalizado & Local & Hombre \\
\hline TE-19 j & Educación & Técnico & Maestro & Más 10 años & Voluntario & Local & Hombre \\
\hline $\mathrm{TJ}-11 \mathrm{~b}$ & Justicia & Técnica & $\begin{array}{c}\text { Trabajadora } \\
\text { social }\end{array}$ & Menos 10 años & Regulado & Provincial & Mujer \\
\hline TJ-13 p & Justicia & Técnica & Antropóloga & Menos 10 años & Voluntario & Provincial & Mujer \\
\hline TSa-01 r & Salud & Técnica & Socióloga & Más 10 años & Normalizado & Provincial & Mujer \\
\hline TSa-06 i & Salud & Técnica & Médica & Más 10 años & Normalizado & Provincial & Mujer \\
\hline TSe- $07 \mathrm{p}$ & Seguridad & Policía & Administrativa & Menos 10 años & Regulado & Provincial & Mujer \\
\hline TSe-14 a & Seguridad & Guardia Civil & Abogado & Menos 10 años & Normalizado & Provincial & Hombre \\
\hline TSo-02 r & Social & Técnica & $\begin{array}{c}\text { Trabajadora } \\
\text { social }\end{array}$ & Menos 10 años & Voluntario & Provincial & Mujer \\
\hline TSo-05 a & Social & Técnico & Antropólogo & Menos 10 años & Regulado & Provincial & Hombre \\
\hline
\end{tabular}

\section{Validación de resultados}

Los resultados han sido triangulados por dos investigadores llegando a un consenso acerca de los criterios de agrupación a la hora de la descripción de los mismos. Asimismo, los resultados principales y las conclusiones fueron expuestos en una sesión a los entrevistados, mostrando un nivel de acuerdo total entre los mismos y las opiniones que manifestaron en su día.

\section{Consideraciones éticas}

Se comunicaron verbalmente los objetivos del estudio cuando se realizó la petición de colaboración por vía telefónica o personal. Previamente a la realización de la entrevista se les facilitó a los entrevistados una carta introductoria en la que, más deta- lladamente, se les informaba de las implicaciones del estudio, la institución que lo realizaba y la metodología empleada y se les pedía su consentimiento expreso para la grabación de la entrevista.

\section{RESULTADOS}

\section{Objetivo 1. Explorar las expectativas profesionales, personales, sobre las organizaciones en las que actúan y en cuanto a las poblaciones sobre las que actúan}

El principio de eficiencia y efectividad aparece en el estudio como una de las principales expectativas de los profesionales. Aspiran a crecer personal y profesionalmente trabajando conjuntamente. El hecho de conocerse y generar confianza entre pro- 
Tabla 2

Categorías de análisis

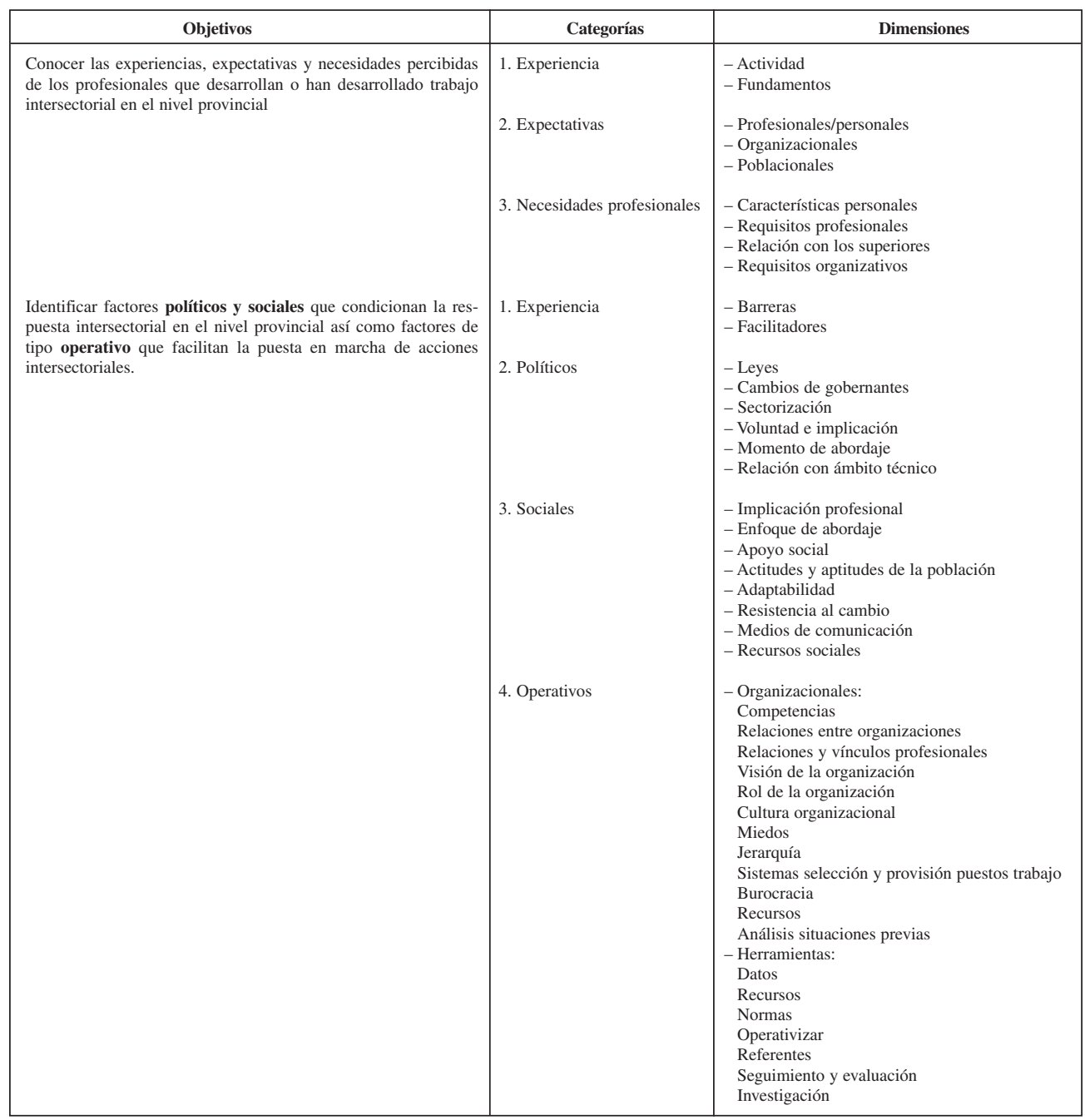

fesionales de distintas organizaciones fomenta el respeto al otro y el conocimiento de sus competencias.

Se explicita la necesidad de que las organizaciones hagan un esfuerzo en dar una única respuesta al ciudadano mejorando, de esta manera, el servicio. Los profesionales de Educación, Salud y Bienestar Social, son los que manifiestan esta necesidad.
Las organizaciones han de utilizar su potencial para generar cambios en la población.

La necesidad de AIS se hace mucho más acuciante en aquellas poblaciones en situación de discapacidad, desventaja social o dependencia y tiene la finalidad de hacer efectivo el principio de equidad. 


\section{Objetivo 2. Explorar los fundamentos que los profesionales encuentran en el desarrollo de estos trabajos}

La AIS se fundamenta en el propio desempeño de los objetivos laborales. En cuanto a esto hay diferencias con respecto a los sectores estudiados: los profesionales de los sectores de educación, salud e igualdad y bienestar social, la consideran una metodología de trabajo inherente a las propias funciones mientras que los profesionales de justicia y seguridad han sido consciente en los últimos tiempos de que su intervención puntual sobre un determinado problema no es suficiente.

El origen de una determinada AIS puede surgir de la aparición de problemas puntuales que pueden dar lugar a la normalización en la colaboración entre organizaciones.

\section{Objetivo 3. Explorar las necesidades de tipo laboral/profesional y de tipo personal que requieren estos trabajos}

Las características identificadas como «personales» por los entrevistados (tales como sensibilidad, sentido común,...) se insertan en los requisitos profesionales para el desempeño de estas acciones. Es necesaria una capacitación a través de formación en metodologías de intersectorialidad y en habilidades sociales y de comunicación y negociación.

Por parte de los superiores se han de establecer relaciones basadas en la confianza y la cesión de autonomía en niveles inferiores.

\section{Objetivo 4. Identificar factores políticos y sociales que condicionan la respuesta intersectorial en el nivel provincial, así como factores de tipo operativo que facilitan la puesta en marcha de acciones intersectoriales}

Se han identificado una serie de necesidades en el ámbito político: asumir e impulsar un marco legal para el abordaje intersectorial de problemas; establecimiento políticas intersectoriales a largo plazo; existencia de ámbitos de decisión compartidos, superando la excesiva sectorización; colocar los intereses de la población general por encima de políticas de confrontación; existencia de una demanda social de respuestas políticas; y objetivos políticos basados en propuestas técnicas.

También se han identificado una serie de características sociales que pueden favorecer u obstaculizar las acciones intersectoriales: los movimientos ciudadanos, de afectados y profesionales pueden ser los determinantes del momento y tipo de abordaje de un problema; el acelerado ritmo de cambio social actual obliga a una readecuación constante en las respuestas dadas; la información y capacitación de poblaciones afectadas; el acercamiento a poblaciones de afectados y de otras culturas, mejora la efectividad; y tener en cuenta la responsabilidad de los medios de comunicación como generadores de opinión.

Por último, a nivel práctico y como posibles instrumentos a poner en marcha por parte de las organizaciones, se identifican los factores operativos que a su vez han sido subdivididos en organizativos y en herramientas:

Organizativos: El miedo a compartir competencias que existe en muchas organizaciones actuales puede ser superado con la existencia de programas de intervención comunes y objetivos conjuntos; el conocimiento y respeto entre organizaciones; la resistencia al cambio que no beneficia las innovaciones; delimitación clara entre los ámbitos político y técnico; implicación de directivos y de la organización en general; las relaciones entre organizaciones han de ser igualitarias no existiendo prepotencia por parte de alguna de ellas; implicación de todos los actores que formen parte del problema y de la respuesta; incorporación de 
agentes que tengan un contacto real con las poblaciones afectadas y con el problema en cuestión; flexibilización del sistema de selección y provisión de puestos de trabajo que permita la adaptación a los objetivos intersectoriales; y el «egoísmo organizativo» o no mirar al entorno descuidando el servicio al ciudadano centrándose en el propio control de la organización.

Herramientas: Sistemas de Información compartidos entre varias organizaciones; marco normativo flexible que se pueda adaptar a las peculiaridades locales; la sujeción al voluntarismo de los profesionales de estas acciones, ha de ser superado con instrumentos que normalicen estos trabajos; introducción de elementos dinamizadores como concreción de objetivos, establecimiento de plazos y existencia de un profesional referente en cada una de las organizaciones implicadas; establecimiento de canales de comunicación continuos y periódicos; incorporación de estrategias para el mantenimiento de acuerdos; y realización de estudios de calidad del propio trabajo.

\section{DISCUSIÓN}

Es indudable que ha habido una progresiva toma de conciencia de la existencia de «los problemas sociales» que, afectando a toda la sociedad, son independientes de la actuación de cada sujeto y que sólo pueden ser resueltos mediante su estudio y la actuación conjunta ${ }^{18}$

Las políticas equitativas, los problemas de desigualdad, requieren una real descentralización del poder e implicación de los actores en cada uno de los niveles del proceso de elaboración de políticas ${ }^{19}$

Este estudio supone un avance y profundización en los condicionantes de la AIS en el contexto andaluz y en el marco de organizaciones de ámbito provincial, algo que es novedoso si se tiene en cuenta que los estudios publicados en España recientemente, tratan de la evaluación de experiencias y acciones locales concluyendo con la identificación de algunos elementos genéricos que condicionan la AIS $^{20-22}$. En este sentido, el Programa de Actividades Comunitarias en Atención Primaria (PACAP) está suponiendo una importante contribución metodológica al trabajo comunitario desde el sector salud ${ }^{23}$. Tal y como advertía Julio Suárez, hay que diferenciar la AIS del trabajo comunitario ${ }^{4}$, entendiéndolo como la última y más importante fase de cualquier acción de este tipo, pero insertado en un proceso más amplio e integral.

Las organizaciones de los sectores de seguridad y justicia se ajustan a una configuración maquinal con la normalización de los procesos de trabajo como mecanismos de coordinación del trabajo; sin embargo, en el resto de sectores, se ejercen presiones para profesionalizar las organizaciones, con objeto de minimizar la influencia que otros, tanto colegas como directivos y cargos políticos, tienen sobre su trabajo. Estas últimas consiguen la coordinación por medio de la normalización de habilidades y conocimientos ${ }^{24}$, haciendo de la formación un parámetro clave en todo trabajo que se llame profesional.

Por otro lado, la creación de equipos de trabajo con carácter permanente o temporal para la solución de problemas determinados entre varias organizaciones de diferentes sectores ha sido uno de los elementos identificados como facilitadores del trabajo intersectorial $^{25}$.

El incremento creciente en el ritmo de cambio social tiene la consecuencia inmediata de una necesidad de continua adaptación de las personas a esa sociedad, adaptación obstaculizada por lo que se ha denominado uniformización y el anonadamiento de la cultura organizativa ${ }^{18}$. La posibilidad de subsistencia de una organización dependerá de su capacidad de adaptación al entorno cambiante en el que está operando ${ }^{24,26}$. 
Las resistencias organizativas al cambio detectadas en este estudio, están relacionadas con la potente cultura de las organizaciones que en sí resulta un bloqueo a esa adaptación que el entorno demanda en las sociedades actuales, en general y en las acciones intersectoriales, en particular ${ }^{27}$. Cualquier intervención a estos niveles, no puede obviar esa cultura, hablándose incluso de su instrumentalización al servicio de los objetivos de la organización ${ }^{28}$.

Una de las grandes limitaciones del estudio es no haber dispuesto de algo más de tiempo para que la investigación hubiese concluido con la realización de un grupo de expertos formado por los profesionales que han participado, y que de ahí se extrajeran las acciones de mejora priorizadas. No obstante, este estudio aporta a las organizaciones una serie de claves que les permitirán identificar, tras un análisis DAFO (debilidades, amenazas, fortalezas, oportunidades), sus puntos fuertes y aquellos pendientes de alguna mejora, si pretenden poner en marcha AIS.

Las expectativas que expresan los profesionales con el desarrollo de una AIS son la mejora del trabajo, el enriquecimiento personal, unificar, mejorar o dar respuesta al ciudadano y garantizar la equidad en poblaciones de discapacitados o en desventaja social.

Se precisan profesionales con una serie de características personales como son el sentido común, la flexibilidad; y con una cualificación y formación integral con capacidad polivalente e independencia y con habilidades en comunicación y negociación.

En la etapa de definición del problema resulta fundamental la presión social previa ejercida que demande respuesta a ese problema que llega a determinar el momento y el tipo de abordaje.

El análisis ha de realizarse con el máximo nivel de consenso social y político implicando a todos los actores y estable- ciendo una continuidad en las políticas que se establezcan.

Características sociales actuales como son el dinamismo y la complejidad de los problemas hacen necesarias estructuras organizativas de tipo orgánico sin excesiva normalización, capaces de adaptarse con mayor facilidad a los cambios del entorno, y descentralizado, por el cuerpo de conocimientos complejo en el que se basa. El ritmo de cambio en las organizaciones es muy lento y está obstaculizado por su cultura.

Desde los sectores educativo, social y de salud se demandan organizaciones en las que el profesional tenga mayor capacidad de decisión y menos control burocrático, con coordinación basada en la normalización de habilidades adquiridas a través de la formación y la interactuación con otras organizaciones.

Elementos organizativos como el miedo a compartir ámbitos de decisión o competencias, la idea de no igualdad y que existan organizaciones que no ganen algo, sistemas de selección y provisión de puestos de trabajo no versátiles; influyen en el desarrollo de la AIS.

La introducción de instrumentos como el establecimiento de canales de comunicación continuos y periódicos, de creación de grupos de trabajo para problemas puntuales en los que se introduzcan elementos dinamizadores como trabajo por programas y objetivos, existencia líderes, compartir sistemas de información y marco normativos flexibles; favorecería estas acciones.

\section{BIBLIOGRAFÍA}

1. Piedrola-Gil G, Rey-Calero J, Domínguez-Carmona M, et al. La salud y sus determinantes. En: Medicina preventiva y salud pública (9 edición) Barcelona: Ediciones Científicas y Técnicas, S.A; 1991.p. 3-10.

2. Salleras Sanmartí, La Educación Sanitaria. Principios, métodos y aplicaciones. Madrid: Díaz de Santos; 1989. 
3. Dahlgren G. Appendix 1: The need for intersectoral action for health. In: Intersectorial action for health. European health Policy Conference: opportunities for the Future. Copenhagen: World health Organization Regional Office for Europe; 1994; .p.11-26

4. Suarez J. Contribución al marco teórico para el desarrollo intersectorial en salud. Educ Med Salud. 1992; 26: 225-41

5. Federal/Provincial/Teritorial Advisory Comité on Population Health. Intersectorial Action Towards Population Health. June; 1999

6. Suarez J. Proposición de un modelo para el estudio y desarrollo de la acción intersectorial en salud. Educ Med Salud. 1993; 27: 61-81

7. Van Herten LM, Rejineveld SA., Gunning-Schepers LJ. Holanda. Rationalising chances of success in intersectorial health policy making. J Epidemiol Community Health. 2001; 55 (5):342-47.

8. Atención Primaria de Salud. Serie Salud Para Todos. No. 1. Ginebra: OMS;1978.

9. Formulación de estrategias con el fin de alcanzar Salud Para Todos en el año 2000. Serie Salud Para Todos. No. 2. Ginebra: OMS; 1979.

10. Barnard K, Ritsatakis A, Svensson P. G. Equity and intersectorial action for health. The role of intersectoral cooperation in combating inequities in health in national strategies for health for all. The Nordic School of Public Health. 1987.

11. Oficina Regional Europea de la OMS. Health 21: an introduction to health for all policy framework for the WHO European Region. European health for All Series, No. 5. Ginebra: OMS;1998.

12. Rodriguez JA, Guillén MF. Organizaciones y profesiones en la sociedad contemporánea. En: REIS 59 (92): 9-18

13. Tercer Plan Andaluz de Salud 2003-2008.Sevilla: Consejería de Salud de la Junta de Andalucía; 2002

14. Priorización e Implantación del Tercer Plan Andaluz de Salud 2003-2008. Sevilla. Consejería de Salud de la Junta de Andalucía; 2004

15. Fernández de Sanmamed Santos. Adecuación de las normas de publicación en revistas científicas a las investigaciones cualitativas. Aten Primaria 2000; 25: 502-4

16. Britten N. Técnicas cualitativas de entrevista en investigación médica. BMJ. 1995;251
17. López-Aranguren E. El análisis de contenido. 2001; 150-158

18. Lucas Marín A. El estudio sociológico de la organización empresarial. En: Sociología para la empresa. Madrid: McGraw Hill/Interamericana de España, SA. p. 3-33.

19. Whitehead M. The concepts and principles of equity and health. Copenhagen: World Health organization Regional Office for Europe. p. 10-16

20. Aranda García E, De Dios M, Ruiz Estalrich MC, Fayos Pérez I., Ferrero Sanchos F, Tomas Dols S, Bueno Cañigral FJ. El sida ese gran reto: una experiencia multidisciplinaria e intersectorial en un municipio del Área Sanitaria 13 de la Comunidad Valenciana. Pub of SEISIDA. 2001; 12 (4): 351-4.

21. Peiró R, López R, Marrodánd J, Fernández C, Ramírez C. Actividades intersectoriales en la prevención de accidentes de tráfico. Gac Sanit 2003;17(4):332-4

22. García Gonzalez, J, Rodríguez Rodríguez P. Rompiendo distancias: Un programa integral para prevenir y atender la dependencia de las personas mayores en el medio rural. Rev Esp Geriatr Gerontol. 2005; 40: 22-33

23. Sociedad Española de Medicina de Familia y Comunitaria. ¿Cómo iniciar un proceso de intervención y participación comunitaria desde un centro de salud? De la reflexión a las primeras intervenciones. Madrid: Instituto de Salud Pública de la Comunidad de Madrid.

24. Mintzberg H. Sobre las organizaciones.. En: Mintzberg y la dirección. Madrid: Díaz de Santos; 1991. p. 107-33.

25. Monzón C. Aportación de la Psicología de la empresa. Los grupos en la empresa. En: Sociología para la empresa. Madrid: McGraw Hill/Interamericana de España; 1994. p. 37-62.

26. Gómez-Llera G. La adaptación de las organizaciones a su entorno. En: Sociología para la empresa. Madrid: McGraw Hill/Interamericana de España. p. 283-300.

27. Berger P, Luckman T. Los fundamentos del conocimiento en la vida cotidiana. En: La construcción social de la realidad. Amorrortu-Murguía;1986.p. 36-52.

28. Clegg SR. Modern Organization. Organization Studies in the Postmodern World, Londres: Sage; 1990. 